\title{
Análise do Aplainamento de Ganho Óptico e da Equalização de Potência em Sistemas WDM
}

\author{
Rafael H. Coradin e Alexandre A. P. Pohl
}

\begin{abstract}
Resumo- Este trabalho apresenta uma análise do desnível de potência e OSNR na recepção de portadoras ópticas transmitidas em um sistema WDM que opera na faixa entre 1529 e $1535 \mathrm{~nm}$. Nas simulações foram utilizadas a técnica de aplainamento de ganho em amplificadores ópticos com o emprego de uma grade de Bragg e a técnica de equalização de potência com o emprego de atenuadores ópticos variáveis. As simulações foram realizadas em ambiente computacional que se assemelha ao test-bed da rede GIGA.

Palavras-Chaves-Amplificadores ópticos, sistemas WDM, aplainamento de ganho, equalização de potência.

Abstract - This work presents an analysis of the power and OSNR imbalance at the reception of optical carriers transmitted over a WDM system operating in the range of 1529 and $1535 \mathrm{~nm}$. In the simulations a technique for gain equalization based upon a fiber Bragg grating and a technique for optical power equalization based on variable optical attenuator were employed. The simulations were performed in a software environment that resembles the test-bed of the GIGA Network.
\end{abstract}

Keywords-Optical amplifiers, WDM systems, gain equalization, power equalization.

\section{INTRODUÇ̃̃O}

Sistemas WDM (Wavelength Division Multiplexing) proporcionaram um enorme impacto no crescimento da capacidade de transmissão em enlaces de comunicação óptica. Esse crescimento foi também em grande parte possível pelo advento da amplificação óptica, mais precisamente pelo desenvolvimento dos amplificadores a fibra dopada com Érbio (EDFA), capazes de amplificar múltiplos comprimentos de onda em uma mesma fibra [1].

$\mathrm{O} E D F A$ convencional, dopado apenas com $\mathrm{Er}^{3+}$, apresenta um perfil de ganho assimétrico na faixa de 1525 a $1560 \mathrm{~nm}$, possuindo um valor de pico em torno do comprimento de onda de $1533 \mathrm{~nm}$, e uma faixa de ganho menor, relativamente plana que se estende aproximadamente de 1542 a $1560 \mathrm{~nm}$. Esse amplificador, quando utilizado em toda essa faixa de ganho, gera um desnivelamento de potência óptica entre portadoras em sistemas multi-canais. Ou seja, portadoras existentes na

Rafael H. Coradin, CITS, Centro Internacional de Tecnologia de Software, R. do Semeador, 702, 81.270-150, Curitiba, PR; Alexandre A. P. Pohl, DAELN/CPGEI, Universidade Tecnológica Federal do Paraná, UTFPR, Av. Sete de Setembro, 3165, 80.230-901 Curitiba, PR. faixa de $1533 \mathrm{~nm}$ são mais amplificadas do que aquelas na região de $1550 \mathrm{~nm}$. As diferenças de ganho podem gerar uma série de efeitos indesejáveis na fibra como fenômenos não lineares [2], que degradam o sinal das portadoras ópticas na recepção. Assim, frequentemente a faixa em torno de $1533 \mathrm{~nm}$ não é utilizada. Contudo, essa mesma faixa pode ser empregada se técnicas de equalização de ganho (denominadas daqui por diante de planificação de ganho) forem introduzidas, isso é, a curva de ganho do amplificador pode ser aplainada, de forma que todos os canais aplicados ao longo da faixa de comprimento de onda em que o amplificador opera tenham o mesmo ganho. Entre os métodos desenvolvidos pode-se citar a utilização de filtros em grades de Bragg [3] e filmes finos [4].

Um outro método que pode ser empregado com a mesma finalidade utiliza um equalizador de potência óptica (EDPO), que pode ser localizado em pontos estratégicos da rede. $\mathrm{O}$ equalizador empregado nas simulações deste trabalho é baseado em um dispositivo desenvolvido para a rede GIGA [5] e que utiliza atenuadores ópticos variáveis (VOAs), sintonizados dinamicamente na simulação, para nivelar as potências. Nas simulações o EDPO é localizado depois do dispositivo de inserção e remoção de comprimentos de onda (OADM - Optical Add-Drop Multiplexer). O estudo apresentado tem como objetivo comparar o desempenho das técnicas de planificação de ganho com grades de Bragg e a técnica que utiliza o EDPO e verificar a planicidade do perfil de potências ópticas que chega ao receptor no final da rede. São apresentados resultados de simulação, que mostram o efeito isolado e combinado dessas duas técnicas aplicadas na faixa de operação do amplificador entre 1529 e $1534 \mathrm{~nm}$, a qual representa a faixa de maior desnível de ganho. Como base para a simulação é utilizado o enlace representado pela Rede GIGA [6], em um modelo computacional com parâmetros e configuração próximas da rede existente e composta por 10 EDFAs.

A Rede GIGA é um enlace experimental destinado à avaliação de dispositivos, protocolos e serviços de redes de comunicação óptica [6]. O enlace liga Campinas ao Rio de Janeiro, passando por várias cidades, em uma distância de aproximadamente $670 \mathrm{~km}$. Nele é empregado um sistema WDM com 8 portadoras ópticas na banda $\mathrm{C}$, cujo espaçamento é de $200 \mathrm{GHz}$. Os oito canais operam atualmente com tráfego de dados até 2,5 Gbps, prevendo-se uma expansão para 16 canais com taxa de $10 \mathrm{Gbps}$.

A Fig. 1 mostra um diagrama lógico simplificado da configuração da Rede GIGA, no qual, para efeitos de simulação, é introduzido um add-drop óptico $(O A D M)$ no site "São Paulo". Para as simulações foi utilizado o pacote de 
software VPI TransmissionMaker ${ }^{\circledR}$ [7], que permite a modelagem de dispositivos e o emprego dos mesmos em um sistema de comunicação óptica. O Rio de Janeiro foi considerado fim de curso e os resultados obtidos na simulação têm esse ponto como sua referência.

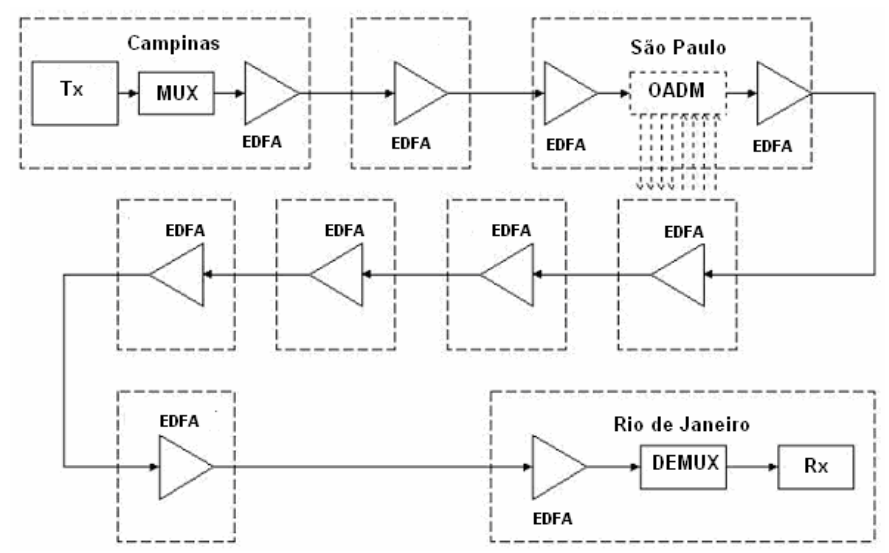

Fig. 1. Diagrama esquemático da Rede GIGA empregada na simulação.

Além das duas técnicas já mencionadas, o estudo foi completado com a introdução de uma técnica para correção das oscilações do ganho geradas devido a perturbações no regime de saturação do amplificador e que podem acarretar na degradação do sinal na recepção. Esse problema pode surgir tanto pela ausência repentina de portadoras em um enlace WDM como pelo chaveamento óptico de portadoras gerado em add-drops e roteadores. As oscilações no ganho em regime saturado têm sido extensivamente tratadas na literatura [8] [9], com soluções que empregam a supressão totalmente óptica ou o controle automático da corrente do laser de bombeamento.

A descrição de como essas técnicas foram usadas no ambiente de software é descrita a seguir.

\section{A. Planificação do Ganho Óptico}

A planificação de ganho foi realizada empregando-se a técnica de filtros a grades de Bragg. A razão dessa escolha deve-se a grande faixa espectral em que atua, a baixa perda por inserção, à facilidade de sua fabricação em fibra óptica e ao seu custo relativamente baixo. Uma grade de Bragg pode ser modelada utilizando o módulo Filter_FBG_TLM_Opt do software VPI, que representa a grade por um filtro com resposta ao impulso infinita. Esta grade tem um perfil de perdas ajustado de maneira a contrapor o perfil de ganho do $E D F A$. Isto é possível ajustando-se alguns dos seus parâmetros como: largura de banda, freqüência central de atuação e rejeição espectral. A grade utilizada na simulação possui os seguintes parâmetros: freqüência central de $1533 \mathrm{~nm}$, largura de banda de 19,6 nm e rejeição espectral de $6 \mathrm{~dB}$. Esse filtro consegue manter o sinal da ASE (Amplified Spontaneous Emission) com um ripple de menos de $1 \mathrm{~dB}$ em um trecho de aproximadamente $30 \mathrm{~nm}$, a partir do comprimento de onda de $1530 \mathrm{~nm}$.

\section{B. Controle de Oscilações no Regime Saturado}

Para o controle das oscilações no ganho dos amplificadores quando está trabalhando em regime saturado, usou-se a técnica de controle de corrente do laser de bombeamento. $\mathrm{O}$ modelo utiliza a técnica de realimentação, usando a potência de saída total do amplificador como base para cálculo da corrente do laser de bombeamento, através de um bloco de controle, que altera a potência do laser de acordo com a necessidade. $\mathrm{O}$ corte dos canais em uma rede simulada utilizando esta técnica causa uma pequena variação instantânea de potência na saída do EDFA, que logo é corrigida pelo sistema de supressão [9].

\section{Equalização de Potência}

Mocki et al [5] estudaram os efeitos da equalização de potência óptica e seu comportamento na Rede GIGA. Neste trabalho foi sugerida uma configuração com a utilização de um Equalizador de Potências Ópticas (EDPO) localizado após o quarto $E D F A$ e outro localizado após último $E D F A$ da rede mostrada na Fig. 1. O EDPO projetado e construído é baseado no método de equalização de potência utilizando VOAs. O sistema consiste em um dispositivo onde os canais são individualmente separados por meio de um demultiplexador. A potência é então direcionada a um conjunto de fotodetectores, no qual o sinal sofre conversão para o domínio elétrico. Em um bloco de processamento eletrônico os valores de potência são armazenados e comparados por meio de uma rotina que aponta o desnível de potência entre os canais. Baseado neste resultado, diferentes tensões são aplicadas aos atenuadores pertencentes ao bloco de entrada e os canais sofrem atenuações proporcionais a sua potência inicial. Um bloco com essas mesmas características foi projetado no VPI e utilizado nas simulações, cujos resultados são apresentados neste trabalho. A descrição desse bloco e seu funcionamento podem ser encontrados em [10].

\section{RESUltados}

As simulações foram realizadas considerando a Rede GIGA composta por 8 portadoras ópticas, mas na faixa de comprimentos de onda de 1528,8 a $1534,3 \mathrm{~nm}$ e com canais espaçados em $0,8 \mathrm{~nm}$, conforme a recomendação G.692 da ITU [11]. As portadoras são lançadas na estação de Campinas com $1 \mathrm{~mW}$ de potência cada, a uma taxa de $10 \mathrm{Gbps}$. Os comprimentos de onda foram escolhidos de maneira a utilizar a faixa do espectro onde o ganho do EDFA apresenta maior variação, próximo a $1530 \mathrm{~nm}$. Durante as simulações foram retiradas no $O A D M$, localizado entre o terceiro e o quarto EDFA, as quatro portadoras de maior comprimento de onda e substituídas por outros quatro canais de mesmo comprimento de onda, mas com um valor de potência maior do que aquelas que adentram o dispositivo. Isto é feito de forma a provocar oscilações de potência na rede tais que retiram o amplificador do regime saturado e forçar situações extremas onde o efeito da equalização torne-se mais perceptível. Os comprimentos de onda das portadoras utilizadas nas simulações são mostrados na Tabela I.

O desnível de potência observado é considerado como sendo a diferença entre a maior e a menor potência medida entre as 8 portadoras na recepção. Como referência, simulou- 
TABELA I

PORTADORAS UTILIZADAS NAS SIMULAÇÕES DA REDE GIGA

\begin{tabular}{ccc} 
Canal & Frequência $(\mathrm{THz})$ & Comprimento de onda $(\mathrm{nm})$ \\
\hline 1 & & 1534,53 \\
2 & 195,5 & 1533,74 \\
3 & 195,6 & 1532,96 \\
4 & 195,7 & 1532,18 \\
5 & 195,8 & 1531,39 \\
6 & 195,9 & 1530,61 \\
7 & 196,0 & 1529,83 \\
8 & 196,1 & 1529,05
\end{tabular}

se inicialmente a rede GIGA sem remoção e substituição de portadoras no $O A D M$, ou seja, as 8 portadoras são lançadas no site Campinas, atravessam os 10 EDFAs e são observadas na recepção, site do Rio de Janeiro. Quatro situações foram consideradas: sem qualquer tipo de equalização ou planificação de ganho (referência), apenas com planificação de ganho por filtros a grade de Bragg adicionados aos EDFAs; apenas com equalização de potência por um EDPO localizado na saída do quarto EDFA (site de São Paulo) e por ambas as técnicas atuando simultaneamente. Essas situações foram simuladas sem o emprego da técnica de controle automático de ganho nos amplificadores. Contudo, na seção E, são apresentados resultados com a inclusão dessa técnica.

\section{A. Potência e OSNR X Comprimento de Onda na Recepção da Rede GIGA - Caso sem Adição/Remoção de Canais}

Esta simulação é de especial interesse para a avaliação do comportamento das técnicas equalização óptica em enlaces estáticos e com EDFAs localizados estrategicamente para a regeneração da potência óptica lançada na entrada da rede. Ela não representa uma situação em que haja variação de potência óptica ao longo do enlace gerada por queda de canais ou por variações devido à presença de $O A D M s$ e roteadores. A Fig. 2 mostra a potência dos 8 canais observada na recepção do sistema para as quatro situações descritas.

No caso em que nenhuma técnica de equalização é aplicada observa-se um desnível de potência entre portadoras da ordem de 27,2 dB. A adição do EDPO em São Paulo não melhora muito essa situação, embora se possa perceber um nivelamento a partir do comprimento de onda de $1532 \mathrm{~nm}$. Porém, ao se aplicar apenas a técnica de planificação de ganho a grades de Bragg obtém-se um espectro quase plano em toda a faixa. Comparando o resultado do emprego dessa técnica de forma isolada com aquela na qual ambas as técnicas são aplicadas simultaneamente, percebe-se que o desnível de potência apresenta uma redução de $92,6 \%$ e $94,1 \%$ respectivamente. Isto se deve ao fato de a rede ser estática, ou seja, não haver a atuação de $O A D M s$. Neste caso, o desnível de potência entre os canais para o caso sem equalização é devido principalmente ao perfil irregular do ganho na região de comprimentos de onda próximos de $1530 \mathrm{~nm}$.

Da mesma forma, a relação sinal ruído óptica $(O S N R)$ varia pouco quando se utiliza a técnica de planificação de ganho apenas. A variação é de $1,1 \mathrm{~dB}$ entre as portadoras em toda a faixa. Esse valor sobe para 1,6 dB quando as duas técnicas são usadas simultaneamente. Esse resultado pode ser observado na Fig. 3.

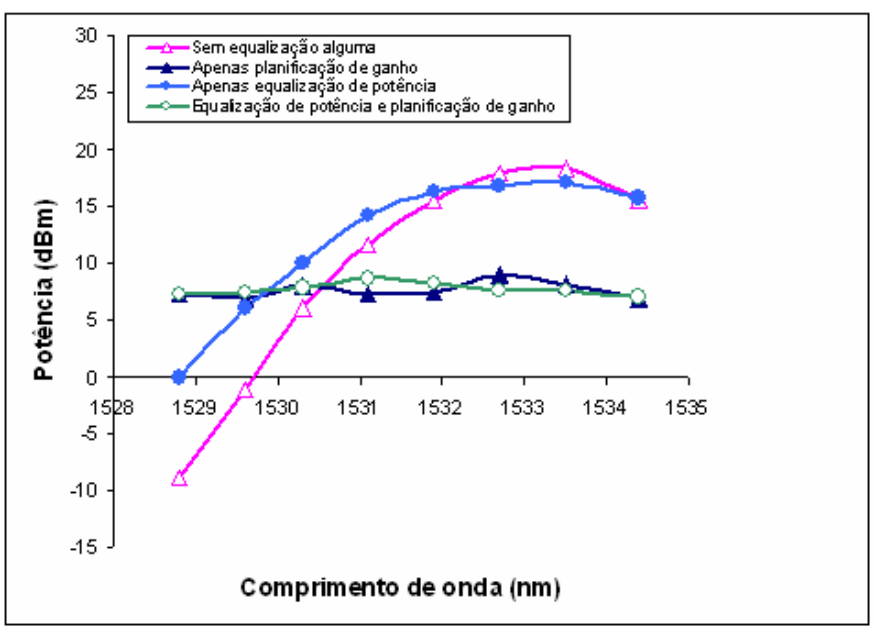

Fig. 2. Potência na recepção da rede GIGA. Caso sem adição/remoção de canais para quatro situações de equalização.

Esses resultados sugerem a utilização apenas da técnica de planificação do ganho óptico usando grades de Bragg em configurações de rede onde não há variações de potência (rede estática).

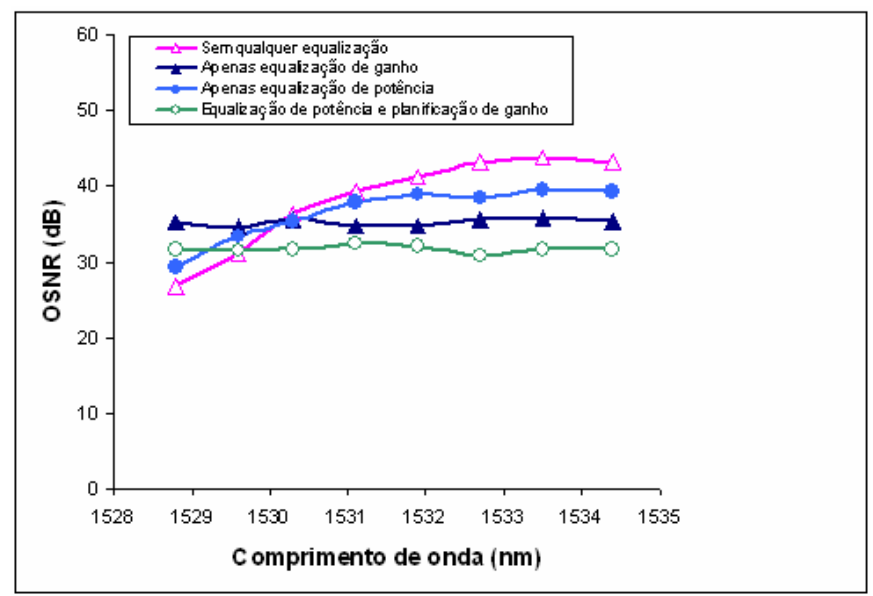

Fig. 3. OSNR na recepção da rede GIGA. Caso sem adição/remoção de canais para quatro situações de equalização

\section{B. Potência e OSNR X Comprimento de Onda na Recepção da Rede GIGA - Caso com Adição/Remoção de Canais}

A potência de cada portadora na recepção do sistema para as mesmas quatro situações tratadas na seção $\mathrm{B}$ é mostrada na Fig. 4. Contudo, neste caso, se utiliza o $O A D M$. Para essa simulação, os quatro canais de menor comprimento de onda foram retirados e substituídos por outros quatro no $O A D M$, com o mesmo comprimento de onda, porém inseridos com 1 $\mathrm{mW}$ de potência por portadora no site de São Paulo.

No caso de um sistema sem qualquer tipo de equalização, os 4 canais substituídos são devolvidos ao sistema com uma potência menor do que os que são mantidos. Como não é feito qualquer tipo de equalização ao longo do enlace, as portadoras 
chegam à recepção com um claro desnível entre as quatro primeiras e as quatro últimas, cujo valor é da ordem de $20 \mathrm{~dB}$. Ao se utilizar o EDPO na saída do site onde atua o $O A D M$, as quatro portadoras com comprimentos de onda mais baixos são equalizadas logo após sua substituição. Porém essa equalização é pontual (no site São Paulo), mas ao ser amplificadas pelos outros EDFAs antes de chegar à recepção, tornam-se novamente desniveladas ao atravessarem o restante da rede até a recepção. Contudo, o desnível de potência entre elas torna-se menor, como visto na Fig. 4.

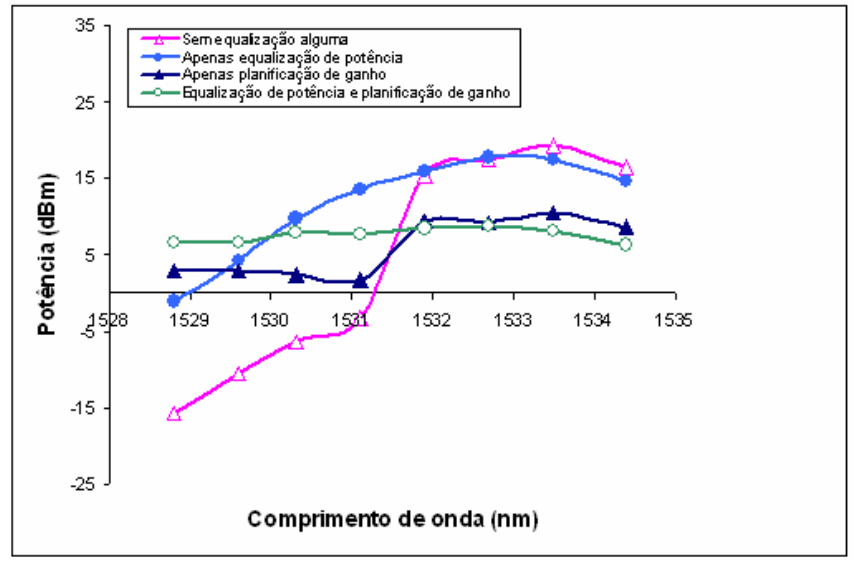

Fig. 4. Potência dos canais na recepção do sistema para quatro situações de equalização.

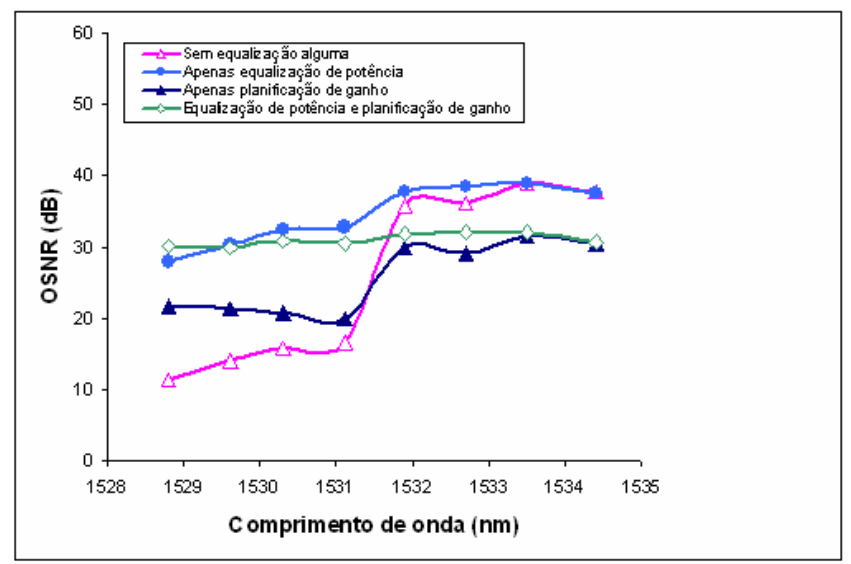

Fig. 5. OSNR dos canais na recepção do sistema para quatro situações de equalização. Casos com substituição de canais de $1 \mathrm{~mW}$ no site de São Paulo através do OADM.

Se apenas a técnica de planificação de ganho é utilizada ao longo da rede, as portadoras com comprimento de onda menor e as quatro portadoras com comprimento maior mantêm-se nos mesmos patamares de potência. Porém o desnível de potência causado pela substituição das quatro portadoras com comprimento de onda menor no site de São Paulo não pode ser revertida. Neste caso, apenas a atuação das duas técnicas em conjunto consegue fazer com que a variação temporal, devido à remoção e adição de canais, e à variação espacial das potências desenvolvida ao longo da rede pelo cascateamento de EDFAs, possam ser reduzidas. Neste caso, as portadoras apresentam um desnível de potência na recepção da ordem de $2,5 \mathrm{~dB}$.

Com relação à OSNR, o comportamento das portadoras é semelhante ao que o corre com as potências ópticas, como pode ser visto na Fig. 5. As quatro portadoras de menor comprimento de onda, substituídas no site de São Paulo, apresentam potência menor que os demais e, portanto, sua OSNR também será menor. Isto faz com que em um sistema sem equalização haja um desnível significativo, da ordem de $20 \mathrm{~dB}$, entre elas nesse ponto da rede. Com a atuação do EDPO, realiza-se uma equalização temporal desse desnível. Mas apenas a utilização simultânea de ambas as técnicas faz com que o desnível de OSNR seja menor, apresentando uma diferença de 2,2 dB no final da rede.

\section{Desnível de Potência X Distância - Rede GIGA sem Adição/Remoção de Canais}

O desnível de potência entre os canais deve-se manter estável, não só na recepção, mas ao longo de toda a rede, para evitar que erros na transmissão dos dados ocorram em pontos intermediários e sejam propagados até a recepção desses.

O comportamento do desnível de potência ao longo de toda a extensão da Rede GIGA foi simulado para as quatro situações já descritas e os resultados mostrados na Fig. 6. As curvas mostram o comportamento para o caso A.

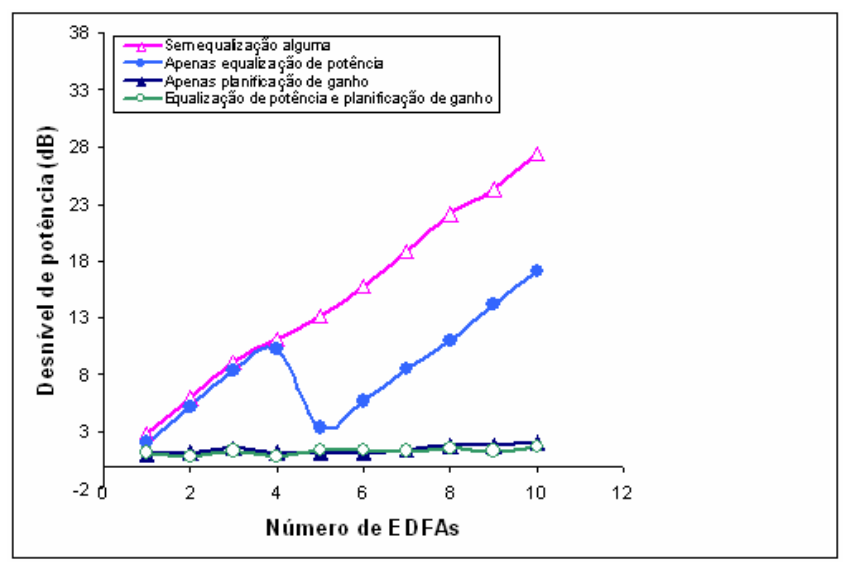

Fig. 6. Desnível de potência em função do número de EDFAs que as portadoras ópticas atravessam na Rede GIGA. Caso sem atuação do OADM.

Observa-se da análise dos resultados da Fig. 6, que o desnível de potência entre as portadoras aumenta linearmente ao longo da extensão rede para a situação sem qualquer equalização. Este fato se deve basicamente ao perfil irregular de ganho em todos os EDFAs. A utilização do EDPO no site de São Paulo permite a redução desse desnível, porém, ela é pontual, se restringindo a este site. Logo após atravessarem o EDFA seguinte o desnível começa a aumentar novamente, como pode ser observado da curva que representa este caso.

A aplicação da técnica de planificação de ganho a grades Bragg em cada EDFA da rede mostra-se, então, como a alternativa mais eficiente, pois consegue fazer com que o desnível de potência entre as portadoras mantenha-se num 
patamar constante em toda a rede. A aplicação conjunta das duas técnicas pouco modifica o comportamento do desnível de potência ao longo da rede nesse caso.

\section{Desnível de potência X Distância - Rede GIGA com Adição/Remoção de Canais}

A Fig. 7 mostra como o desnível de potência se comporta ao longo da Rede GIGA para o caso B.

De forma semelhante ao que ocorre no caso C, o desnível de potência aumenta à medida que mais EDFAs são cascateados quando não há uma técnica de equalização sendo aplicada. Aqui, porém, este aumento é ainda maior no site de São Paulo quando quatro dos canais são substituídos e os canais que os substituem possuem uma potência menor que os demais. Este salto no valor do desnível de potência (ver curva com triângulos vazios na Fig. 7) pode ser contornado com a introdução da técnica de equalização de potência, como pode ser observado na curva representada por círculos cheios. $\mathrm{Na}$ saída do quarto EDFA é utilizado o EDPO, e o salto do desnível antes observado na curva representada por triângulos vazios (sem equalização) não acontece. A deficiência da utilização do EDPO é que sua atuação se restringe ao ponto onde está localizado na rede e o desnível volta a crescer no restante do trecho percorrido pelos canais, mas com valores menores do que o caso onde não há aplicação de quaisquer técnicas.

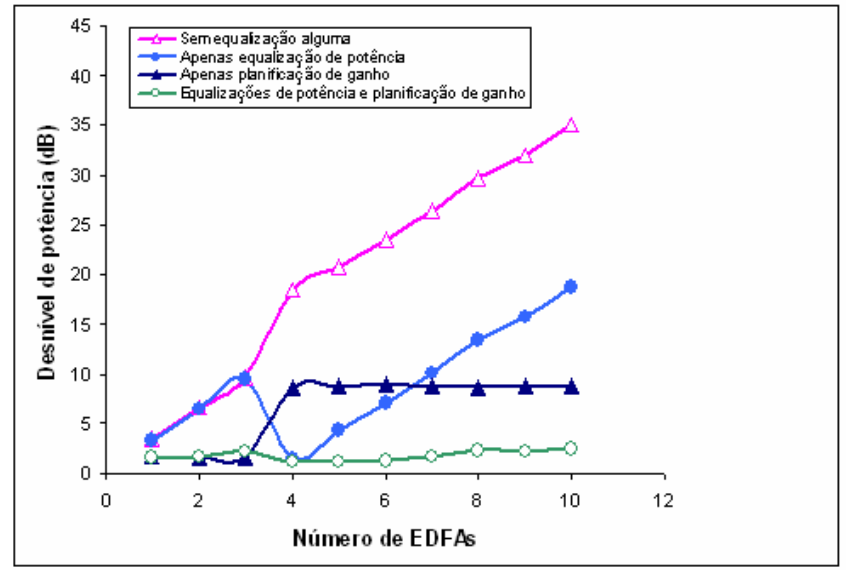

Fig. 7. Evolução do desnível de potência na Rede GIGA em função da distância. Caso onde 4 canais de $1 \mathrm{~mW}$ são substituídos no site de São Paulo

A técnica de planificação de ganho com grades de Bragg consegue evitar que o desnível de potência entre os canais cresça indefinidamente com a quantidade de EDFAs, pois mantém o ganho de todos os canais praticamente igual ao longo da rede. Porém, como se observa na curva representada por pontos triangulares cheios na Fig. 7, uma vez desequalizados os canais, a técnica com grades de Bragg não consegue corrigir esta diferença. É o que ocorre no site São Paulo, onde a potência das quatro portadoras substituídas difere da potência dos canais remanescentes e a técnica de planificação apenas manterá esta diferença no trecho restante em que as portadoras tiverem que se propagar.
Somente a utilização das técnicas de planificação de ganho e de equalização de potência atuando em conjunto consegue, então, agregar as propriedades de ambas, mantendo o desnível de potência praticamente constante ao longo de todo o enlace. Enquanto a utilização do EDPO equaliza os canais quando alguns deles são substituídos por outros de potência desigual, as grades de Bragg integradas aos EDFAs evitam com que haja um crescimento em cascata desse desnível ao longo da rede em decorrência do perfil de ganho irregular dos amplificadores.

\section{E. Controle de Oscilações no Ganho de Amplificadores}

De maneira a verificar o desempenho da melhor técnica para manter a equalização entre os canais na recepção de um sistema com efeitos de variação na potência de entrada acentuados, simulou-se a Rede GIGA em uma situação onde um dos canais é retirado, e não há qualquer substituição deste. Porém, a técnica baseada no controle de corrente do laser de bombeio foi introduzida e estrategicamente colocada no EDFA do site de São Paulo, onde o efeito das oscilações é mais acentuado, pois o canal com menor comprimento de onda é removido causando uma variação brusca na potência de entrada do EDFA. O papel do controle é, então, de compensar a mudança no valor do ganho causado por esta variação.

Quatro situações foram simuladas: sem qualquer tipo de equalização, apenas com planificação de ganho a grades de Bragg, com planificação de ganho e equalização de potência (EDPO) e com planificação de ganho e supressão de oscilações no ganho. O canal com menor comprimento de onda foi removido no site de São Paulo. A potência dos canais na recepção do sistema para essas quatro situações é mostrada na Fig 8.

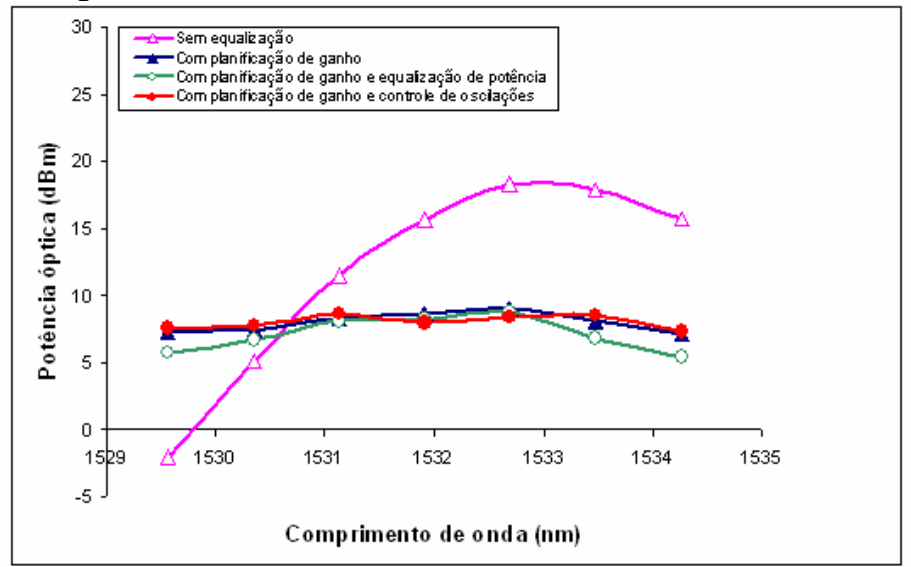

Fig. 8. Potência óptica dos 7 canais remanescentes na recepção da rede GIGA para quatro situações descritas na seção $\mathrm{E}$.

Para o caso sem qualquer tipo de equalização, os canais 5 e 6 têm uma amplificação maior que os demais, isto por estarem situados na região espectral próxima do pico de ganho do EDFA. Ao longo da rede essa diferença no ganho se acumula, gerando um desnível de potência entre os canais que chega a 20,3 dB na recepção. Contribui ainda para o aumento desse desnivelamento o fato do ganho óptico do EDFA ter um aumento quando da retirada do canal 8, no site de São Paulo. É devido a esse desnivelamento que se utilizou aqui a técnica 
de controle de ganho do amplificador através do controle da corrente do laser de bombeamento.

O uso da técnica de equalização de potência permite apenas uma equalização temporal, mas espacialmente é pouco eficiente. O filtro a grade de Bragg é o elemento que pode auxiliar no nivelamento das potências dos canais ao longo da rede. Mas é apenas com as técnicas de planificação de ganho e controle automático de ganho atuando em conjunto que os valores de desnível de potência podem ser otimizados. A redução chega a ser de $20,3 \mathrm{~dB}$, para o caso sem qualquer técnica de equalização atuando, para 1,3 dB nesse último caso.

\section{CONCLUSÃo}

Foi simulado o comportamento de 8 portadoras ópticas na região de comprimentos de onda onde a variação do ganho dos EDFAs é maior (próximo à $1530 \mathrm{~nm}$ ) no ambiente computacional que reproduz a Rede GIGA. Para a simulação onde não foram adicionados ou removidos canais, o desnível de potência entre estes canais melhorou de $27,17 \mathrm{~dB}$, no caso sem equalização de potência ou planificação do ganho, para $1,65 \mathrm{~dB}$ no caso onde há planificação de ganho por filtros a grades de Bragg e equalização de potência por EDPO atuando em conjunto. A utilização de equalizadores de ganho associados aos EDFAs proporciona um desnível de potência e OSNR praticamente constante ao longo de todo o enlace. Para os casos sem $O A D M$, nota-se que o desnível de potência, em nenhum ponto da rede foi superior a $2,03 \mathrm{~dB}$ e o de $O S N R$, não foi maior que $1,13 \mathrm{~dB}$ para o caso onde há apenas planificação de ganho atuando. Esses resultados se contrapõem aos valores de 2,94 dB a 27,34 dB para o desnível de potência, e de $1,26 \mathrm{~dB}$ a $16,98 \mathrm{~dB}$ para o desnível de $O S N R$, no caso onde não há qualquer método de controle do ganho e da potência de saída do EDFA.

Uma avaliação do desnível de potências ópticas para uma montagem com um único EDFA foi realizada por Fugihara et al [12]. Nesse trabalho, 20 canais foram lançados na região do espectro de maior ganho dos EDFAs e o desnível obtido com uma única grade de Bragg foi de $2 \mathrm{~dB}$.

Observando-se os casos de uma rede dinâmica, ou seja, com atuação de um $O A D M$, percebe-se que apenas a planificação de ganho não é suficiente. Enquanto que no caso sem $O A D M$ a atuação conjunta da planificação de ganho e equalização de potência causou uma melhora do desnível de potência na recepção de 2,03 dB para 1,65 dB apenas e o desnível de OSNR aumentou de $1,13 \mathrm{~dB}$ para $1,56 \mathrm{~dB}$. No caso com remoção e substituição de canais por outros de $1 \mathrm{~mW}$ de potência no site de São Paulo a melhora foi de $11,30 \mathrm{~dB}$ para 2,49 dB para o desnível de potência e de 11,40 dB para 2,20 dB para o desnível de $O S N R$.

Nenhuma outra avaliação do desnível de potências e $O S N R$ de canais ópticos lançados na janela dos $1530 \mathrm{~nm}$ em um sistema dinâmico com EDFAs cascateados foi encontrado na literatura.

Foi simulado ainda um sistema onde há apenas portadoras retiradas, mas sem inserção de outras, ao longo da Rede GIGA de maneira a verificar-se o efeito das oscilações na potência de entrada do amplificador atuando no regime saturado. A utilização de uma técnica baseada no controle da corrente do laser de bombeio atuando em conjunto com a técnica de planificação de ganho, otimizou o desnível de potência. O valor do desnível entre as sete portadoras remanescentes diminuiu de 20,3 dB no caso sem qualquer tipo de equalização para $1,3 \mathrm{~dB}$.

Os resultados apresentados neste trabalho apontam para utilização de técnicas diferentes, dependendo das características do enlace, para contornar os efeitos degradantes na transmissão de portadoras ópticas em sistemas WDM operando na faixa de $1530 \mathrm{~nm}$. Para enlaces estáticos sugerese a utilização de uma técnica de planificação de ganho apenas. Para redes onde há a substituição de portadoras, no entanto, a sugestão é da utilização de uma técnica de planificação de ganho em conjunto com uma técnica para equalização de potência reduzindo os desníveis temporais e espectrais do sinal. E, finalmente, para sistemas onde ocorre variações na potência total e que alteram o regime de operação do amplificador, como ocorre no desligamento de portadoras ao longo da rede, a sugestão é utilizar uma técnica de controle automático de ganho, estrategicamente posicionada, de modo a suprimir essas oscilações, e uma técnica de planificação de ganho para manter o patamar das potências das portadoras constante em toda sua extensão.

\section{REFERÊNCIAS}

[1] Sun, Y., Srivastava, A. K., Zhou, J., Sulhoff, J. W. "Optical fiber amplifiers for WDM optical networks", Bell Labs Technical Journal, pp.187-206, January-March, 1999.

[2] Chraplyvy, A. R. "Limitations on Lightwave Communications Imposed by Optical-Fiber Nonlinearities". IEEE Journal of Lightwave Technology, v. 8, n. 10, p. 1548-1556, October, 1990

[3] Guy, M., Lauzon, J., Rochette, M., Trépanier, F., "Applications of Bragg gratings in optical amplifiers". Proc. SPIE v. 4087, p. 411, November, 2000.

[4] Mimura, Y., Mizuno, K., Yoshitaka, I., Masuda, K., Itoh, T., Nishi, Y., "Gain-flattening filters using dielectric multilayer thin film", Furukawa Review, No. 21, pp. 1-5, 2002.

[5] Mocki Jr, A., Kalinowski, H. J., Pohl, A. A. P., "Design and Simulation of an In-Line Optical Gain Equalizer for Application in WDM Systems", IEEE ICTON 221 Th.B1.4, pp. 221-224, 2005

[6] Scarabucci, R. R., Stanton, M. A., de Barros, M. R. X., Salvador, M. R., Rossi, S. M., Simoes, F. D., Rocha, M. L., da Silva Neto, I. L., Rosolem, J. B., Fudoli, T. R. T., Mendes, J. M. D., Castro, N. F., Machado, I., Reggiani, A. E., Paradisi, A., Martins, L., "Project GIGA-high-speed experimental IP/WDM network, Testbeds and Research Infrastructures for the Development of Networks and Communities, 2005". Tridentcom 2005. First International Conference on, p. 242-251, February, 2005.

[7] VPI Photonics, VPI Photonics website, disponível em: www.vpiphotonics.com. Acesso em 03 de agosto de 2007.

[8] Oliveira, Julio Cesar Rodrigues Fernandes; Floridia, Claudio; Rossi, Sandro Marcelo; Rosolem, J. B.; Bordonalli, Aldario C. "Evaluation of Transient Dynamic Impact in Gain Controlled EDFAs". In: International Telecommunication Symposium - ITS, 2006, Fortaleza, 2006.

[9] Pantoja, A. P., Véliz, R. O., "Estrategias de control de transientes en cascada de EDFAs empleando estabilización de la ganancia mediante efecto laser", X Congresso Internacional de Telecomunicaciones SENACITEL, 2002.

[10] Coradin, R. H., "Estudo e Simulação de Técnicas de Planificação de Ganho Óptico e Equalização de Potência em Redes WDM". Dissertação apresentada ao CPGEI, Centro de Pós Graduação em Engenharia e Informática, UTFPR, 2007.

[11] ITU-T Rec. G.692: Optical interfaces for multichannel systems with optical amplifiers, 1999. Disponível em : http://www.itu.int/.

[12] Fugihara, M. C., Kalinowski, H. J., André, P. S., "Estudos para Equalização de Ganho em um AFDE com uma única Rede de Bragg", pp. 1-4, X Simpósio Brasileiro de Micro-Ondas e Optoeletrônica, 2002. 